Ferrata Storti Foundation

\title{
Characterization of breakthrough hemolysis events observed in the phase III randomized studies of ravulizumab versus eculizumab in adults with paroxysmal nocturnal hemoglobinuria
}

Haematologica 2021

Volume 106(1):230-237

\section{Correspondence:}

ROBERT A. BRODSKY

rbrodsky@jhmi.edu

Received: September 3, 2019.

Accepted: January 9, 2020.

Pre-published: January 16, 2020.

https://doi.org/10.3324/haematol.2019.236877

(C)2021 Ferrata Storti Foundation

Material published in Haematologica is covered by copyright. All rights are reserved to the Ferrata Storti Foundation. Use of published material is allowed under the following terms and conditions:

https://creativecommons.org/licenses/by-nc/4.0/legalcode. Copies of published material are allowed for personal or internal use. Sharing published material for non-commercial purposes is subject to the following conditions:

https://creativecommons.org/licenses/by-nc/4.0/legalcode, sect. 3. Reproducing and sharing published material for commercial purposes is not allowed without permission in writing from the publisher.
Robert A. Brodsky, ${ }^{1}$ Régis Peffault de Latour, ${ }^{2,3}$ Scott T. Rottinghaus, ${ }^{4}$ Alexander Röth, ${ }^{5}$ Antonio M. Risitano, ${ }^{6}$ Ilene C. Weitz, ${ }^{7}$ Peter Hillmen, ${ }^{8}$ Jaroslaw P. Maciejewski, ${ }^{9}$ Jeff Szer, ${ }^{10}$ Jong Wook Lee, ${ }^{11}$

Austin G. Kulasekararaj, ${ }^{12}$ Lori Volles, ${ }^{4 a}$ Andrew I. Damokosh, ${ }^{4}$ Stephan Ortiz, ${ }^{4}$ Lori Shafner, ${ }^{4 b}$ Peng Liu, ${ }^{4}$ Anita Hill ${ }^{8 c}$ and Hubert Schrezenmeier ${ }^{13,14}$

${ }^{1}$ Division of Hematology, Department of Medicine, Johns Hopkins University School of Medicine, Baltimore, MD, USA; ${ }^{2}$ Université Paris Diderot, Paris, France; ${ }^{3}$ French Reference Center for Aplastic Anemia and PNH Hematology-Bone Marrow Transplantation, Hôpital Saint-Louis AP-HP, Paris, France; ${ }^{4}$ Alexion Pharmaceuticals Inc., Boston, MA, USA; ${ }^{5}$ Department of Hematology, West German Cancer Center, University Hospital Essen, University of Duisburg-Essen, Essen, Germany; ${ }^{6}$ Hematology, Department of Clinical Medicine and Surgery, Federico II University of Naples, Naples, Italy; ${ }^{7} J a n e$ Anne Nohl Division of Hematology, Keck-USC School of Medicine, Los Angeles, CA, USA; ${ }^{8}$ Department of Haematology, St James's University Hospital, Leeds, UK; ' Department of Translational Hematology and Oncology Research, Taussig Cancer Institute, Cleveland Clinic Foundation, Cleveland, OH, USA; ${ }^{10} \mathrm{Clinical}$ Haematology, Royal Melbourne Hospital, Melbourne, Victoria, Australia; ${ }^{11}$ Department of Hematology, Seoul St. Mary's Hospital, College of Medicine, The Catholic University of Korea, Seoul, Republic of Korea; ${ }^{12}$ Department of Haematological Medicine, King's College Hospital, NIHR/Wellcome King's Clinical Research Facility, London, UK; ${ }^{13}$ Institute for Clinical Transfusion Medicine and Immunogenetics, German Red Cross Blood Transfusion Service Baden-Württemberg-Hessen, Germany and University Hospital UIm, UIm, Germany and ${ }^{14}$ Institute of Transfusion Medicine, University of UIm, UIm, Germany

${ }^{a}$ Current address: Principal, Volles Consulting Group, Old Lyme, CT, USA.

${ }^{\circ}$ Current address: Vista Life Innovations, Madison, CT, USA.

'Current address: Alexion Pharmaceuticals, Inc., Boston, MA, USA.

\section{ABSTRACT}

E culizumab is first-line treatment for paroxysmal nocturnal hemoglobinuria (PNH); however, approximately $11-27 \%$ of patients may experience breakthrough hemolysis (BTH) on approved doses of eculizumab. Ravulizumab, a new long-acting C5 inhibitor with a four times longer mean half-life than eculizumab, provides immediate, complete, and sustained C5 inhibition over 8-week dosing intervals. In two phase III studies, ravulizumab was non-inferior to eculizumab $(P$ $\leq 0.0004$ ) for the BTH endpoint; fewer patients experienced BTH with ravulizumab versus eculizumab in both studies (301 [complement inhibitor-naïve patients], $4.0 \%$ vs. $10.7 \%$; 302 [patients stabilized on eculizumab at baseline], $0 \%$ vs. $5.1 \%$ ). In the current analysis, patientlevel data were evaluated to assess causes and clinical parameters associated with incidents of BTH reported during the 26 -week treatment periods in the ravulizumab phase III PNH studies. Of the five BTH events occurring in ravulizumab-treated patients across the studies, none were temporally associated with suboptimal C5 inhibition (free C5 $\geq 0.5$ $\mu \mathrm{g} / \mathrm{mL})$; four $(80 \%)$ were temporally associated with complement-amplifying conditions (CAC). Of the 22 events occurring in eculizumab-treated patients, 11 were temporally associated with suboptimal C5 inhibition, including three events also associated with concomitant infection. Six events were associated with CAC only. Five events were unrelated to free C5 elevation or reported CAC. These results suggest that the immediate, 
complete, and sustained C5 inhibition achieved through weight-based dosing of ravulizumab reduces the risk of BTH by eliminating BTH associated with suboptimal C5 inhibition in patients with $\mathrm{PNH}$. (Registered at clinicaltrials.gov identifiers: Study 301, NCT02946463; Study 302, NCT03056040.)

\section{Introduction}

Until recently, eculizumab was the only approved treatment for paroxysmal nocturnal hemoglobinuria $(\mathrm{PNH}){ }^{1,2}$ and since its regulatory approval in 2007 , it has changed the paradigm for the treatment of patients with $\mathrm{PNH}^{3 ., 4}$ Longterm experience has established that eculizumab is efficacious and well tolerated; however, approximately $11-27 \%$ of patients may experience breakthrough hemolysis (BTH) on approved dosages of eculizumab during long-term treatment..$^{5.7}$

Breakthrough hemolysis, characterized by the return of intravascular hemolysis and reappearance of classical PNH symptoms, ${ }^{5,8-11}$ may occur due to suboptimal C5 inhibition ${ }^{1}$ and/or CAC such as infection, surgery, or pregnancy that may lead to increased complement activation resulting from higher $\mathrm{C} 3 \mathrm{~b}$ density. ${ }^{12-14}$ In some patients with suboptimal C5 inhibition or CAC, BTH may be ameliorated by shortening the 2-week dosing interval and/or increasing the dose of eculizumab.,7

Ravulizumab is a new long-acting C5 inhibitor developed to reduce the treatment burden associated with eculizumab through an improved dosing regimen and is now approved for treatment of adult patients with PNH in the USA, Japan, and Europe. ${ }^{11,15-17}$ It is notable that the mean terminal halflife of ravulizumab is approximately four times longer than that of eculizumab, allowing ravulizumab to provide immediate, complete, and sustained terminal C5 inhibition with an 8-week dosing interval. ${ }^{11,15-17}$

In the two largest international phase III clinical studies conducted to date in PNH patients, ravulizumab was noninferior to eculizumab across all key efficacy endpoints in patients naïve to complement inhibitor therapy (study 301) as well as in those on stable eculizumab therapy (study 302). ${ }^{16,17} \mathrm{BTH}$, a key secondary endpoint in both studies, was defined as one or more new or worsening symptoms or signs of intravascular hemolysis (fatigue, hemoglobinuria, abdominal pain, dyspnea, anemia [hemoglobin $<10$ $\mathrm{g} / \mathrm{dL}$ ], major adverse vascular event [MAVE] including thrombosis, dysphagia, or erectile dysfunction) in the presence of elevated lactate dehydrogenase (LDH) $\geq 2$ times the upper limit of normal (ULN) after prior LDH reduction to $<1.5 x U L N$ while on therapy. Point estimates for proportions of patients with BTH favored ravulizumab in study $301(4.0 \%$ vs. $10.7 \%$, difference, $-6.7 \%$ [ $95 \%$ confidence interval (CI): $\left.-14.21,0.18] ; P_{* i}<0.0001\right)$ and study $302(0 \%$ vs. $5.1 \%$, difference, $-5.1 \%$ [95\% CI: $-8.89,18.99 ; P_{\text {w }}$ $<0.0004) .{ }^{16,17}$ The purpose of the current analysis was to investigate the causes of and clinical parameters associated with incidents of BTH at the patient level in both studies. Post hoc analyses were also performed to further evaluate $\mathrm{BTH}$ in ravulizumab- and eculizumab-treated patients.

\section{Methods}

\section{Patients and treatment}

Both studies (study 301 and 302) included patients aged $\geq 18$ years with a confirmed diagnosis of PNH by flow cytometry. ${ }^{16,17}$
In study 301 (clinicaltrials.gov identifier: NCT02946463), adult patients naïve to complement inhibitor with $\mathrm{LDH} \geq 1.5$ times the ULN and at least one PNH symptom (consistent with high disease activity as described in the Summary of Product Characteristics ${ }^{2}$ ) were randomized 1:1 to receive ravulizumab or eculizumab for 183 days. ${ }^{17}$ In study 302 (clinicaltrials.gov identifier: NCT03056040), adult patients with $\mathrm{PNH}$ stable on eculizumab therapy (LDH $<1.5$ times ULN) for at least 6 months were randomized 1:1 to ravulizumab or eculizumab for 183 days. ${ }^{16}$ Patients randomized to ravulizumab received loading followed by weight-based dosing every 8 weeks. ${ }^{16,17}$ Patients randomized to eculizumab received $900 \mathrm{mg}$ every two weeks. ${ }^{16,17}$ In study 301, patients with a hemoglobin level $\leq 7 \mathrm{~g} / \mathrm{dL}$ or $\leq 9 \mathrm{~g} / \mathrm{dL}$ in the presence of anemia-related signs or symptoms warranting transfusion received red blood cell transfusion. ${ }^{17}$ Data from patients who experienced BTH in the 26week study period were subject to detailed investigation of BTH events. Both studies were approved by the institutional review board or independent ethics committee at participating centers and were conducted in accordance with the Declaration of Helsinki and the Council for International Organizations of Medical Sciences International Ethical Guidelines.

\section{Derivation of breakthrough hemolysis definition}

Because there is no consensus in the medical literature regarding the definition of $\mathrm{BTH}$, the definition was derived prospectively for use in both studies based on a literature review and interviews of eight study investigators. The resulting definition ${ }^{18}$ was reviewed with and agreed upon by the US Food and Drug Administration, the European Medicines Agency, and the Japan Pharmaceuticals and Medical Device Agency before studies started.

Literature review - the literature review revealed that several biomarkers have been used to describe BTH (Online Supplementary Table S1). Elevated LDH level was most commonly used;, $, 14,19-22$ however, variables such as eculizumab level, ${ }^{20-23}$ other markers of serum hemolytic activity, ${ }^{5,7}$ aspartate aminotransferase level, ${ }^{6}$ reticulocyte count, ${ }^{24}$ and bilirubin level ${ }^{24}$ have also been used. There was no consensus on whether hemoglobin levels and/or transfusion requirements should be considered when defining BTH. ${ }^{7,14,19,21,24}$

Physician interviews - parameters used by participating investigators to measure BTH included symptomatic manifestations (e.g., hemoglobinuria, abdominal pain, and other PNH-related symptoms) and laboratory indicators (e.g., decreased hemoglobin levels, elevated LDH levels, increased total complement levels, and increased bilirubin levels) (Online Supplementary Table S2).

\section{Outcomes}

The key outcome of interest in this study was BTH causality. BTH events were categorized as the following: (i) temporal association that is free C5-related, defined as BTH associated with time-matched occurrence of free C5 $\geq 0.5 \mu \mathrm{g} / \mathrm{mL} ;{ }^{1}$ (ii) CAC-related, defined as BTH due to an inciting event (e.g., infection, trauma, or surgery); or (iii) BTH unrelated to elevated C5 and without a reported time-matched CAC. BTH causality was also analyzed by assessment of the correlation between serum free C5 concentrations $<0.5 \mu \mathrm{g} / \mathrm{mL}$ or $\geq 0.5 \mu \mathrm{g} / \mathrm{mL}$ and BTH.

\section{Additional analyses}

Additional post hoc analyses were conducted as follows: (i) 
Table 1. Incidence of breakthrough hemolysis and overall temporal association.

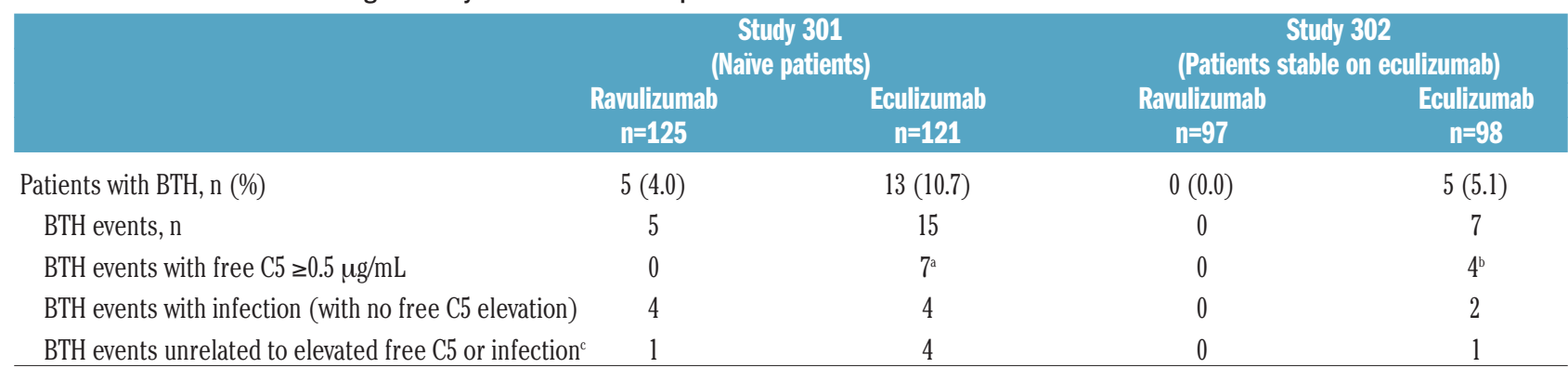

n: total number of patients in treatment group; BTH: breakthrough hemolysis. ${ }^{2}$ Two patients in the eculizumab group with suboptimal C5 inhibition also had concomitant infection. 'One patient in the eculizumab group with suboptimal C5 inhibition also had concomitant infection. ${ }^{\text {These }}$ cases had neither suboptimal C5 inhibition nor concomitant infection identified to explain cause of breakthrough hemolysis.

estimated prevalence of $\mathrm{LDH}$ excursion as reflected by elevations in $\mathrm{LDH}$ to $\geq 2 \mathrm{xULN}$ without regard for signs or symptoms of hemolysis; (ii) estimated exposure-adjusted incidence of BTH per 100 patient-years of study drug exposure; (iii) estimated time to first BTH events temporally associated with suboptimal C5 inhibition with adjustment for competing risk due to CAC or undetermined causality; and (iv) correlation between serum free C5 concentrations $<0.5 \mu \mathrm{g} / \mathrm{mL}$ or $\geq 0.5 \mu \mathrm{g} / \mathrm{mL}$ and BTH events. (See Online Supplementary Appendix for further details of the methods used.)

\section{Results}

\section{Patients}

Study 301 included 125 patients treated with weightbased dosing of ravulizumab and 121 patients treated with approved eculizumab dose $(900$ mg every 2 weeks, q2w), and study 302 included 97 patients treated with weight-based dosing of ravulizumab and 98 patients treated with eculizumab $(900 \mathrm{mg}, \mathrm{q} 2 \mathrm{w}){ }^{16,17}$ We have previously reported patient demographics and clinical characteristics at baseline for both studies. Briefly, the percentage of male patients was $54.5 \%$ and $50.3 \%$ and the mean (standard deviation [SD]) age at first infusion of study drug was 45.5 (15.7) years and 47.7 (14.2) years in studies 301 and 302, respectively. ${ }^{16,17}$ Mean LDH (SD) was 1,606.4 (752.7) U/L and 231.6 (49.2) U/L in study 301 and study 302 , respectively. ${ }^{16,17}$ In study $301,13.8 \%$ of patients had baseline LDH 1.5 to $<3 \times$ ULN and $86.2 \%$ of patients had baseline LDH $\geq 3 x U L N .{ }^{17}$ Mean (SD) glycosylphosphatidylinositol-deficient granulocyte clone size was $84.7 \%(20 \%)$ in study 301 and $83.3 \%(22.5 \%)$ in study $302 .{ }^{16,17}$ Mean (SD) total $\mathrm{PNH}$ red blood cell clone size was $38.6 \%(23.4 \%)$ in study 301 and $60.1 \%$ (31.9\%) in study $302 .{ }^{16,17}$ Mean (SD) hemoglobin levels in study 301 were 9.4 (1.5) g/dL in the ravulizumab arm and 9.6 (1.7) $\mathrm{g} / \mathrm{dL}$ in the eculizumab arm, and in study 302 were 11.1 (1.8) g/dL in the ravulizumab arm and 10.9 (1.8) $\mathrm{g} / \mathrm{dL}$ in the eculizumab arm.

\section{Incidence of breakthrough hemolysis}

As previously reported, there were numerically fewer occurrences of BTH in patients treated with ravulizumab compared with eculizumab in both studies. ${ }^{16,17}$ BTH recurred in the eculizumab but not the ravulizumab arms of both studies: two of the 13 patients experiencing BTH suffered two events each in the 301 study, and in study 302 one of five patients experiencing BTH suffered three events. In study 301, it was noted that the mean (SD) baseline LDH was numerically higher in patients who experienced BTH (1,764.1 [809.7]) as compared with those who did not $(1,593.9$ [748.5]).

\section{Breakthrough hemolysis causality: temporal association}

In study 301, none of the five BTH events in the ravulizumab group were temporally associated with suboptimal C5 inhibition (free C $5 \geq 0.5 \mu \mathrm{g} / \mathrm{mL}$ ) (Table 1); four were temporally associated with CAC (all infections), and one did not have concomitant free C $5 \geq 0.5 \mu \mathrm{g} / \mathrm{mL}$ or a time-matched CAC reported. In the eculizumab group, seven of the 15 BTH events were temporally associated with suboptimal C5 inhibition; CAC (all infections) were associated with six of the 15 events, including two events in patients who also had suboptimal C5 inhibition, and four patients did not have concomitant free C5 $\geq 0.5$ $\mu \mathrm{g} / \mathrm{mL}$ or a time-matched CAC reported.

In study 302, no BTH events were observed in patients treated with ravulizumab. Four of the seven events that occurred in the eculizumab group were temporally associated with suboptimal C5 inhibition, and three events were associated with CAC (all infections), including one event in a patient who also had suboptimal C5 inhibition (Table 1). This patient had three BTH events spanning 113 days of treatment. On day 113, the patient was hospitalized for BTH with symptoms of vomiting, flu-like symptoms, and cola-colored urine; the vomiting was thought to have been caused by a viral infection. This patient discontinued treatment and left the study due to lack of efficacy. One event was neither associated with incomplete C5 inhibition nor a reported CAC.

\section{Breakthrough hemolysis: patient narratives}

Patient narratives on BTH in study 301 are shown in Table 2 for the ravulizumab group and in Table 3 for the eculizumab group. BTH events occurring in the eculizumab group in study 302 are summarized in Table 4. Overall, the majority of patients with BTH $(70 \%$ [16 of 23]) exhibited one or two PNH-related signs or symptoms; the most commonly reported $\mathrm{PNH}$-related signs and symptoms were anemia, dyspnea, hemoglobinuria, and fatigue. With respect to red blood cell transfusion, 8 patients ( 3 in the ravulizumab group and 5 in the eculizumab group) experiencing BTH required transfusion in study 301 (Tables 2 and 3 ) and 3 patients experiencing $\mathrm{BTH}$ in the eculizumab group required transfusion in study 302 (Table 4). 
Table 2. Ravulizumab breakthrough hemolysis events and narratives: study 301.

\begin{tabular}{|c|c|c|c|c|c|c|c|c|}
\hline Pt & $\begin{array}{c}\text { Patients' } \\
\text { characteristics } \\
\text { (sex, age, } \\
\text { body weight) }\end{array}$ & $\begin{array}{l}\text { Breakthrough } \\
\text { hemolysis } \\
\text { event; } \\
\text { symptoms }\end{array}$ & $\begin{array}{l}\text { Study } \\
\text { day }\end{array}$ & $\begin{array}{l}\text { LDHa } \\
\text { (U/L) }\end{array}$ & $\begin{array}{l}\text { Free C5b } \\
\text { ( } \mu g / m L)\end{array}$ & $\begin{array}{c}\text { RBC } \\
\text { transfusion (U) }\end{array}$ & $\begin{array}{l}\text { Possible } \\
\text { CAC }\end{array}$ & Association \\
\hline 1 & $\begin{array}{l}\text { Female; } 34 \mathrm{y} ; \\
\quad 115 \mathrm{~kg}\end{array}$ & $\begin{array}{c}1^{\text {st: fatigue }} \\
\text { abdominal pain, } \\
\text { dyspnea }\end{array}$ & $\begin{array}{l}155 \\
169\end{array}$ & $\begin{array}{l}593 \\
511\end{array}$ & $\begin{array}{l}0.105 \\
0.101\end{array}$ & None & Giardiasis & CAC \\
\hline 2 & $\begin{array}{l}\text { Female; } 30 \mathrm{y} ; \\
\quad 57 \mathrm{~kg}\end{array}$ & $1^{\text {st; }}$ hemoglobinuria & 71 & 687 & 0.0787 & None & Viral infection & $\mathrm{CAC}$ \\
\hline 3 & $\begin{array}{l}\text { Female; } 24 \text { y; } \\
\quad 57 \mathrm{~kg}\end{array}$ & $\begin{array}{l}1^{\text {st; }} \text { hemoglobinuria, } \\
\text { anemia }\end{array}$ & $\begin{array}{l}113 \\
127 \\
155 \\
169 \\
183\end{array}$ & $\begin{array}{l}517 \\
773 \\
513 \\
926 \\
555\end{array}$ & $\begin{array}{l}0.0602 \\
0.0768 \\
0.0428 \\
0.0895 \\
0.0909\end{array}$ & 2 & $\begin{array}{l}\text { Influenza, } \\
\text { upper respiratory infection }\end{array}$ & $\mathrm{CAC}$ \\
\hline 4 & $\begin{array}{l}\text { Male; } 37 \mathrm{y} \\
\quad 66 \mathrm{~kg}\end{array}$ & $1^{\text {st }} ;$ anemia & $\begin{array}{l}71 \\
85 \\
99\end{array}$ & $\begin{array}{l}544 \\
525 \\
827\end{array}$ & $\begin{array}{l}0.0623 \\
0.0414 \\
0.0505\end{array}$ & 2 & Gum infection & CAC \\
\hline 5 & $\begin{array}{l}\text { Male; } 43 \mathrm{y} \\
\quad 70 \mathrm{~kg}\end{array}$ & $1^{\text {st: }}$ anemia & 99 & 615 & 0.0766 & 3 & None & Unexplained \\
\hline
\end{tabular}

C5: complement component 5; CAC: complement-amplifying condition; LDH: lactate dehydrogenase; Pt: patient; RBC: red blood cell; U: number of units transfused; y: years. ${ }^{a}$ The upper limit of normal for LDH is $246 \mathrm{U} / \mathrm{L}$. ${ }^{\circ}$ Free C5 concentrations were quantified using a Gyros-based fluorescence assay.

\section{Additional analyses}

Lactate dehydrogenase excursion only - an analysis assessing the prevalence of excursions of $\mathrm{LDH} \geq 2 \mathrm{xULN}$ after reduction below 1.5xULN without regard to symptoms (i.e., the $\mathrm{LDH}$ portion of the BTH definition) showed treatment with ravulizumab to be superior to eculizumab with significantly fewer ravulizumab-treated patients experiencing $\mathrm{LDH}$ excursions compared with eculizumab-treated patients in both study 301 (8.8\% [95\%CI: 3.8, 13.8] vs. 20.7\% [95\% CI: 13.5, 27.9], respectively; treatment difference $-11.7 \%$ [95\%CI: $-20.7,-2.7] ; P=0.012)$ and study 302 (5.2\% [95\%CI: 0.8, 9.6] vs. 16.3\% [9.0, 23.6], respectively; treatment difference, $-11.2 \%$ [95\% CI: $-20.3,-2.4]$; $P=0.015)$.

Exposure-adjusted BTH event rate - an analysis to evaluate the estimated incidence of BTH events per 100-patient years of exposure showed rates to be approximately 3 -fold higher (incidence rate ratio, 0.32 [95\% CI: 0.11 , 0.92]; $P=0.034)$ in the eculizumab group $(21.5$ [95\% CI: 8.9, 51.7] events per 100 patient-years) versus the ravulizumab group (6.8 [95\% CI: 2.2, 21.5] events per 100 patient-years) in study 301. In study 302, the estimated incidence rate per 100-patient years of exposure was 19.9 (95\% CI: 7.2, 54.9) in the eculizumab group, whereas the exposureadjusted incidence rate was non-estimable in the ravulizumab group because no events were observed.

Time to first event with adjustment for competing risk - in study 301 , the analysis of time to first BTH event due to any cause showed significantly lower hazard in the ravulizumab group compared with the eculizumab group (hazard ratio [HR] $0.36 \quad$ [95\%CI: 0.13, 1.0]; $P=0.049$ ) (Online Supplementary Figure $S 1 A$ ). In the analysis to assess BTH events temporally associated with suboptimal C5 inhibition, after adjustment for competing risk due to a CAC or undetermined causality, the probability of a BTH event due to suboptimal C5 inhibition was shown to be significantly reduced with the ravulizumab group compared with the eculizumab group (HR 0; $P<0.001)$ (Online Supplementary
Figure S2A). In a conservative evaluation, when the same analysis was applied to BTH events temporally associated with suboptimal C5 inhibition or undetermined causality after adjustment for competing risk due to a CAC, the hazard was still significantly lower in the ravulizumab group (HR [95\%Cl]: $0.10 \quad[0.01, \quad 0.81] ; \quad P=0.031$ ) (Online Supplementary Figure S3A). In study 302, the hazard ratio estimates in the time-to-event analyses were all zero due to no BTH events occurring in the ravulizumab group (Online Supplementary Figures $S 1 B, 2 B$ and $3 B$ ).

Correlation of BTH with serum free C5 levels - all post-baseline free $\mathrm{C} 5$ values in ravulizumab-treated patients were $<0.5 \mu \mathrm{g} / \mathrm{mL}$ in both the 301 and 302 studies throughout 26 weeks of treatment. In study 301, the overall percentage of patients with BTH among patients who had any free C5 concentration $\geq 0.5 \mu \mathrm{g} / \mathrm{mL}$ across both treatment groups was $40 \%$ compared with $5.2 \%$ among those who had free C5 concentrations $<0.5 \mu \mathrm{g} / \mathrm{mL}$ for every assessment (relative risk, 7.7) (Online Supplementary Table S3). In study 302, the percentage of patients with BTH among patients who had any free C5 concentration $\geq 0.5 \mu \mathrm{g} / \mathrm{mL}$ was $28.6 \%$ compared with $1.6 \%$ in patients with all free C5 concentrations $<0.5 \mu \mathrm{g} / \mathrm{mL}$ (relative risk, 17.9) (Online Supplementary Table S3).

\section{Discussion}

In patients with $\mathrm{PNH}$ receiving complement inhibitor therapy, a BTH event represents loss of disease control. $\mathrm{BTH}$ is manifested by classical PNH symptoms and can require blood transfusion, ${ }^{12,14,22}$ but more critically it can be associated with the return of the morbidity associated with $\mathrm{PNH}$, including potentially life-threatening thromboembolic events. ${ }^{25-27}$ A consensus definition of BTH derived after a literature review and interviews with $\mathrm{PNH}$ experts, and prospectively accepted by regulatory authorities, was used in the two phase III studies of ravulizumab 
Table 3. Eculizumab breakthrough hemolysis events and narratives: study 301.

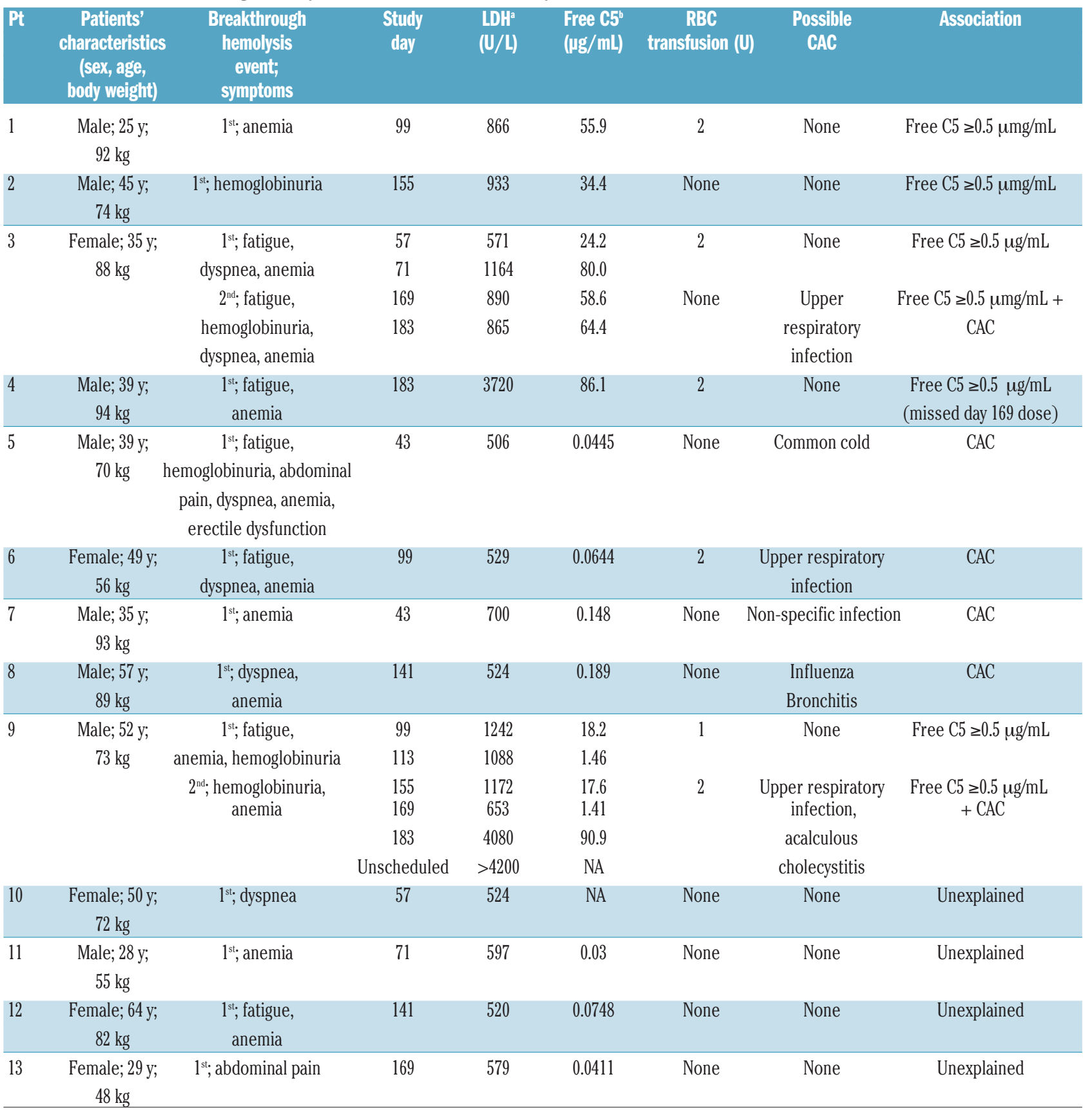

C5: complement component 5; CAC: complement-amplifying condition;LDH: lactate dehydrogenase; NA: not available; Pt: patient; RBC: red blood cell; U: number of units transfused; y: years. ${ }^{a}$ The upper limit of normal for LDH is $246 \mathrm{U} / \mathrm{L}$. ${ }^{\circ}$ Free $\mathrm{C} 5$ concentrations were quantified using electrochemiluminescence ligand binding assay.

to evaluate the incidence and causality of BTH in patients who were naïve to complement inhibitor therapy as well as those who were previously stabilized on eculizumab. ${ }^{18}$ In both studies, ravulizumab was shown to be non-inferior to eculizumab on the assessment of $\mathrm{BTH}_{1},{ }_{1,17}$ and treatment with ravulizumab was associated with numerically fewer episodes of BTH compared with eculizumab in each of these studies.

The current analysis showed that no BTH events in patients treated with weight-based dosing of ravulizumab were associated with elevations in free C5 levels. In contrast, several patients treated with eculizumab across both studies had BTH events that were temporally associated with elevations in free C5 levels (C5 $\geq 0.5 \mu \mathrm{g} / \mathrm{mL})$, suggesting these patients may have had suboptimal C5 control. In such cases, it has been shown that BTH can be successfully managed in some patients by adjusting the dosing amount and/or frequency of eculizumab administration that differs from the approved regimen of eculizumab. ${ }^{5,7,12}$ However, in both studies (301 and 302), patients required maintenance with the approved eculizumab dosing (900 mg every 2 weeks); no changes to the dose levels or dosing regimen were permitted during the study, and if any such changes were made, these patients were excluded from the studies.

Correspondingly, regardless of the treatment group, risk 
Table 4. Eculizumab breakthrough hemolysis events and narratives: study 302.

\begin{tabular}{|c|c|c|c|c|c|c|c|c|}
\hline Pt & $\begin{array}{c}\text { Patients' } \\
\text { characteristics } \\
\text { (sex, age, } \\
\text { body weight) }\end{array}$ & $\begin{array}{l}\text { Breakthrough } \\
\text { hemolysis } \\
\text { event; } \\
\text { symptoms }\end{array}$ & $\begin{array}{l}\text { Study } \\
\text { day }\end{array}$ & $\begin{array}{l}\text { LDHa } \\
\text { (U/L) }\end{array}$ & $\begin{array}{l}\text { Free C5 } \\
(\mu \mathrm{g} / \mathrm{mL})\end{array}$ & $\begin{array}{c}\mathrm{RBC} \\
\text { transfusion (U) }\end{array}$ & $\begin{array}{l}\text { Possible } \\
\text { CAC }\end{array}$ & Association \\
\hline \multirow[t]{4}{*}{1} & Male; $29 \mathrm{y}$; & $1^{\text {stt }}$ hemoglobinuria & 29 & 1257 & 24.1 & None & None & Free $\mathrm{C} 5 \geq 0.5 \mu \mathrm{g} / \mathrm{mL}$ \\
\hline & $95 \mathrm{~kg}$ & $2^{\text {nd, }}$ hemoglobinuria & 57 & 1037 & 24.8 & None & None & Free $\mathrm{C} 5 \geq 0.5 \mu \mathrm{g} / \mathrm{mL}$ \\
\hline & & $3^{\text {rd }}$ hemoglobinuria & 99 & 811 & 19.3 & 1 & None & Free $\mathrm{C} 5 \geq 0.5 \mu \mathrm{g} / \mathrm{mL}$ \\
\hline & & & 113 & 3846 & 91.9 & & & \\
\hline
\end{tabular}

\begin{tabular}{|c|c|c|c|c|c|c|c|c|}
\hline 2 & $\begin{array}{l}\text { Male; } 34 \text { y; } \\
\quad 71 \mathrm{~kg}\end{array}$ & $1^{\text {st }}$ hemoglobinuria & 141 & 618 & 0.1 & None & $\begin{array}{c}\text { Flu-like } \\
\text { symptoms }\end{array}$ & $\mathrm{CAC}$ \\
\hline 3 & $\begin{array}{l}\text { Female; } 47 \mathrm{y} ; \\
75 \mathrm{~kg}\end{array}$ & $\begin{array}{c}\text { 1st; fatigue, } \\
\text { hemoglobinuria, }\end{array}$ & 176 & 515 & 0.1 & 4 & $\begin{array}{c}\text { Acute } \\
\text { pyelonephritis }\end{array}$ & $\mathrm{CAC}$ \\
\hline
\end{tabular}

\begin{tabular}{|c|c|c|c|c|c|c|c|c|}
\hline 4 & $\begin{array}{c}\text { Male; } 60 \mathrm{y} ; \\
79 \mathrm{~kg}\end{array}$ & $\begin{array}{c}1^{\text {st }} \text { fatigue, } \\
\text { hemoglobinuria, } \\
\text { anemia }\end{array}$ & 127 & 1846 & 2.1 & 2 & Gastroenteritis & Free $\mathrm{C} 5 \geq 0.5 \mu \mathrm{g} / \mathrm{mL}+\mathrm{CAC}$ \\
\hline 5 & $\begin{array}{l}\text { Male; } 60 \mathrm{y} ; \\
\quad 84 \mathrm{~kg}\end{array}$ & $\begin{array}{c}1^{\text {st }} \text {, fatigue, } \\
\text { dyspnea }\end{array}$ & 155 & 799 & 0.1 & None & None & Unexplained \\
\hline
\end{tabular}

C5: complement component 5; CAC: complement-amplifying condition; LDH: lactate dehydrogenase; Pt: patient; RBC: red blood cell; U: number of units transfused; y: year. ${ }^{a}$ The upper limit of normal for LDH is $246 \mathrm{U} / \mathrm{L}$. ' $F r e e ~ C 5$ concentrations were quantified using electrochemiluminescence ligand binding assay.

of BTH was approximately 8 -fold ( $40 \%$ vs. $5 \%$ in study $301)$ and 18 -fold (29\% vs. $2 \%$ in study 302$)$ higher in patients with free $\mathrm{C} 5 \geq 0.5 \mu \mathrm{g} / \mathrm{mL}$ compared with those who had free C5 concentrations $<0.5 \mu \mathrm{g} / \mathrm{mL}$ for every assessment. These results suggest that reduction of free C5 may be associated with reduced risk of BTH in patients with $\mathrm{PNH}$.

In study 301 (complement inhibitor-naïve patients), similar proportions of patients in each treatment group experienced infection-related BTH (ravulizumab, 3.2\% [4 of 125]; eculizumab, 3.3\% [4 of 121]), possibly due to proximal complement activation. There was one patient in the ravulizumab group and four patients in the eculizumab group who had adequate C5 inhibition and no apparent infection or other CAC reported by the investigators. In study 302 (patients stabilized on eculizumab at baseline), no ravulizumab-treated patients and $2.0 \%$ ( 2 of 98) of eculizumab-treated patients experienced infectionrelated BTH. One eculizumab-treated patient had a BTH event that was not attributed to suboptimal C5 inhibition or an identifiable CAC.

Observations from these studies suggest that BTH can occur due to infections despite the fact that the terminal complement activity is suppressed. The causes of hemolysis in the setting of infection/sepsis have not been fully elucidated. ${ }^{28}$ It has been shown that exposure of host red blood cells to infectious pathogen cells can cause hemolysis independent of complement activity, suggesting that the complement system may not be the sole cause of infection-triggered hemolysis. ${ }^{13,28}$

Results from the additional analyses of BTH supported and extended observations from the primary analyses. If only the objective LDH component of the BTH definition was considered, a significantly lower proportion of ravulizumab-treated patients experienced BTH compared with eculizumab in both studies. Treatment with ravulizumab was also shown to be associated with a 3 -fold lower exposure-adjusted incidence of BTH events than eculizumab (6.8 vs. 21.5 events per 100 patient-years of exposure), and a $90 \%$ lower risk of BTH due to suboptimal C5 inhibition compared with eculizumab (HR, 0.10; $P=0.031$ ) in study 301. Data in study 302 also support lower risk of BTH with no event occurring in the ravulizumab group.

A unique aspect of these two studies is the consensus definition of BTH. Although not all potential biomarkers and/or causative factors were utilized in the definition of BTH that was used in these phase III studies (e.g., reduction of hemoglobin levels, elevation in reticulocyte count, subtherapeutic serum levels of complement inhibitor), the definition is conservatively based on objective criteria (LDH levels) and well-known, easily identifiable PNHrelated signs and symptoms (e.g., anemia, hemoglobinuria, fatigue, dyspnea), which may facilitate early recognition and treatment of BTH in patients with $\mathrm{PNH}$ receiving complement inhibitor therapy.

Some limitations to this analysis are worthy of note. Approximately $15 \%$ of the observed BTH events were due to unexplained causes (unrelated to insufficient C5 inhibition and without a recognized/reported CAC). These events may have been caused by unrecognized or unreported CAC or other etiological factors. Notably, none of the BTH events in these studies were associated with major adverse vascular events (MAVE). However, this is not surprising since the incidence of MAVE was low $(1.6 \%[n=2]$ and $0.8 \%[n=1]$ for ravulizumab and eculizumab, respectively, in study 301, and no patients experienced MAVE in study 302). Given the importance of BTH in the potential development of associated thrombosis, rigorous clinical interrogation may reveal other and/or new factors associated with manifestations of BTH. Considering the conservative approach for the additional analyses of the BTH data from these studies, where events due to suboptimal C5 inhibition were pooled with undetermined causality and then adjusted for competing risks of CAC, it is likely that the significant between-group differences observed in BTH were driven by treatment-related effects on inhibition of free C5 concentrations. Due to 
the strict definition of BTH applied in these studies, patients were required to have both high $\mathrm{LDH}$ levels and one or more new or worsening signs/symptoms of intravascular hemolysis. Because symptom reporting is at least in part subjective, it is possible the results reported herein could be confounded by ascertainment bias associated with under-reporting of BTH. For example, PNHrelated symptoms may have been unreported due to individual patient or cultural norms, or the symptoms were not associated with severe anemia or hemoglobinuria. This hypothesis is strengthened by the higher number of patients in both treatment arms who experienced LDH elevations but did not have symptoms of BTH reported by investigators. On the other hand, it is possible that patients with infection might have elevated $\mathrm{LDH}$ due to the infection ${ }^{29-31}$ rather than intravascular hemolysis, thereby overestimating the frequency of hemolysis associated with infections.

In summary, weight-based dosing of ravulizumab administered every 8 weeks was associated with numerically fewer episodes of BTH versus eculizumab administered $900 \mathrm{mg}$ every 2 weeks over 26 weeks of complement inhibitor therapy in $\mathrm{PNH}$ patients with high disease activity. The observed differences in BTH rates for ravulizumab versus eculizumab may be attributable to the ability of ravulizumab to completely inhibit free C5 over the entire 8-week dosing interval. Furthermore, no BTH events in the ravulizumab group were associated with free C5 concentrations $\geq 0.5 \mu \mathrm{g} / \mathrm{mL}$. In contrast, some patients treated with eculizumab experienced multiple BTH events that were temporally associated with elevations in free C5 (one resulting in hospitalization). Similar numbers of patients receiving ravulizumab or eculizumab experienced CAC-related BTH, possibly due to proximal complement activation. Overall, results from these two studies demonstrate that ravulizumab achieved immediate and complete inhibition of free C5 over the entire 26-week treatment period, reducing the overall risk of $\mathrm{BTH}$ by eliminating free C5-associated BTH.

\section{Disclosures}

$R A B$ is a member of the scientific advisory board for and receives grant funding from Alexion Pharmaceuticals, Inc.; RPdL has received honoraria, consulting fees, and research support from Alexion Pharmaceuticals, Inc., Pfizer, and Novartis, and has received research support from Amgen; STR is an employee and stockholder of Alexion Pharmaceuticals, Inc.; AR has received honoraria and consulting fees from Alexion Pharmaceuticals, Inc., Apellis Pharmaceuticals, Novartis, and Roche; AMR has received research support, honoraria, and consulting fees from Alexion Pharmaceuticals, Inc., Novartis, Alnylam, and $R a$ Pharma, lecture fees from Alexion Pharmaceuticals, Inc., Novartis, Pfizer, and Jazz, and served as an advisory board member for Alexion Pharmaceuticals, Inc.,
Novartis, Pfizer, and Jazz, as well as consultant for Amyndas; $I C W$ has received honoraria and consulting fees from Alexion Pharmaceuticals, Inc.; $P H$ has received honoraria from and has been a consultant for Alexion Pharmaceuticals, Inc., and has received research support (to the University of Leeds) from Apellis Pharmaceuticals; JPM has received consulting fees from Alexion Pharmaceuticals, Inc., Apellis Pharmaceuticals, and Ra Pharma; has also received speaker fees from and is a member of the Executive Committee of the International PNH Registry for Alexion Pharmaceuticals, Inc.; JS has received research support (to Royal Melbourne Hospital), honoraria, consulting fees, and travel support from Alexion Pharmaceuticals, Inc.; JWL has received honoraria, consulting fees, and research support (to Seoul St. Mary's Hospital) from Alexion Pharmaceuticals, Inc.; $A G K$ has received honoraria, travel support, and consulting fees from Alexion Pharmaceuticals, Inc.; $L V$ is an employee and stockholder of Alexion Pharmaceuticals, Inc.; AID was an employee and stockholder of Alexion Pharmaceuticals, Inc. at the time of the study analysis and during the development of this manuscript; SO is an employee and stockholder of Alexion Pharmaceuticals, Inc.; LS was an employee and stockholder of Alexion Pharmaceuticals, Inc. at the time of this analysis and during development of the manuscript; PLis an employee and stockholder of Alexion Pharmaceuticals, Inc.; AH has received honoraria and consulting fees from Alexion Pharmaceuticals, Inc.; HS has received honoraria and research support (both to University of Ulm) from Alexion Pharmaceuticals, Inc.

\section{Contributions}

Study design: $A H, A I D, A R, P H, H S, R P L, J W L, A K, L S, L V$, STR. Study investigator: $A H, A R, R A B, R P L, A M R, I C W, P H$, $J P M, J S, J W L, A K, H S$. Enrolled patients: $A H, A R, R A B, R P L$, $A M R, I C W, P H, J P M, J S, J W L, A K, H S$. Collection and assembly of data: All authors. Data analysis: AR, AID, HS, PL, SO, STR, RAB. Data interpretation: All authors. Manuscript review and revisions: All authors. Final approval of manuscript: All authors.

\section{Acknowledgments}

The sponsor and investigators would like to thank the patients and their families for their participation in and support for these clinical studies. The authors thank Rodrigo Pavani and Masayo Ogawa of Alexion Pharmaceuticals, Inc. for their contribution to the implementation of the 301 and 302 studies. The authors would like to acknowledge Michael D. Morren, RPh, MBA, of Peloton Advantage, LLC, an OPEN Health Company, and Jennifer M. Kulak, PhD, of ApotheCom (Yardley, PA, USA), who provided editorial and medical writing support funded by Alexion Pharmaceuticals, Inc. Editorial review was also provided by Kenneth Pomerantz, PhD, Shweta Rane, PhD, CMPP, and Radha Narayan, PhD, of Alexion Pharmaceuticals, Inc.

\section{Funding}

This study was supported by Alexion Pharmaceuticals, Inc.

\section{References}

1. SOLIRIS ${ }^{\circledR}$ (eculizumab) injection, for intravenous use. Boston, MA, USA: Alexion Pharmaceuticals Inc. 06/2019.

2. Soliris (eculizumab). [Summary of product characteristics] Paris, France: Alexion Europe SAS, 2018.

3. Griffin M, Munir T. Management of throm- bosis in paroxysmal nocturnal hemoglobinuria: a clinician's guide. Ther Adv Hematol. 2017;8(3):119-126.

4. Hill A, DeZern AE, Kinoshita T, Brodsky RA. Paroxysmal nocturnal haemoglobinuria. Nat Rev Dis Primers. 2017;3:17028.

5. Hillmen P, Muus P, Röth A, et al. Long-term safety and efficacy of sustained eculizumab treatment in patients with paroxysmal nocturnal haemoglobinuria. Br J Haematol.
2013:162(1):62-73.

6. Nakayama H, Usuki K, Echizen H, Ogawa R, Orii T. Eculizumab dosing intervals longer than 17 days may be associated with greater risk of breakthrough hemolysis in patients with paroxysmal nocturnal hemoglobinuria. Biol Pharm Bull. 2016;39(2):285-288.

7. Peffault de Latour R, Fremeaux-Bacchi V, Porcher $\mathrm{R}$, et al. Assessing complement blockade in patients with paroxysmal noc- 
turnal hemoglobinuria receiving eculizumab. Blood. 2015;125(5):775-783

8. Almeida AM, Bedrosian C, Cole A, et al. Clinical benefit of eculizumab in patients with no transfusion history in the International Paroxysmal Nocturnal Haemoglobinuria Registry. Intern Med J. 2017;47(9):1026-1034.

9. Höchsmann B, Sicre de Fontbrune F, Lee JW, et al. Effect of eculizumab in paroxysmal nocturnal hemoglobinuria $(\mathrm{PNH})$ patients with or without high disease activity: results from the International PNH Registry. Haematologica. 2017;102(Suppl 2):188-189.

10. Lee JW, Peffault de Latour R, Brodsky RA, et al. Efficacy of eculizumab in patients with paroxysmal nocturnal hemoglobinuria (PNH) and high disease activity with or without history of aplastic anemia in the International PNH Registry. Blood. 2017; 130(Suppl 1):3487.

11. Röth A, Rottinghaus ST, Hill A, et al. Ravulizumab (ALXN1210) in patients with paroxysmal nocturnal hemoglobinuria: results of 2 phase $1 \mathrm{~b} / 2$ studies. Blood Adv. 2018;2(17):2176-2185.

12. Brodsky RA. Eculizumab: another breakthrough. Blood. 2017;129(8):922-923.

13. Harder MJ, Kuhn N, Schrezenmeier H, et al. Incomplete inhibition by eculizumab: mechanistic evidence for residual C5 activity during strong complement activation. Blood. 2017;129(8):970-980.

14. Miyasaka N, Miura $O$, Kawaguchi $T$, et al. Pregnancy outcomes of patients with paroxysmal nocturnal hemoglobinuria treated with eculizumab: a Japanese experience and updated review. Int J Hematol. 2016; 103(6):703-712

15. ULTOMIRISTM (ravulizumab-cwvz) injec- tion, for intravenous use. Boston, MA, USA: Alexion Pharmaceuticals Inc., 12/2018.

16. Kulasekararaj AG, Hill A, Rottinghaus ST, et al. Ravulizumab (ALXN1210) vs eculizumab in C5-inhibitor-experienced adult patients with PNH: the 302 study. Blood. 2019;133(6):540-549.

17. Lee JW, Sicre de Fontbrune F, Lee LWL, et al. Ravulizumab (ALXN1210) vs eculizumab in adult patients with $\mathrm{PNH}$ naive to complement inhibitors: the 301 study. Blood. 2019, 133(6):530-539.

18. Brodsky RA, De Latour RP, Rottinghaus ST, et al. A prospective analysis of breakthrough hemolysis in 2 phase 3 randomized studies of ravulizumab (ALXN1210) versus eculizumab in adults with paroxysmal nocturnal hemoglobinuria. Blood. 2018; 132(Suppl 1):2330.

19. DeZern AE, Dorr D, Brodsky RA. Predictors of hemoglobin response to eculizumab therapy in paroxysmal nocturnal hemoglobinuria. Eur J Haematol. 2013;90(1):16-24.

20. Hill A, Hillmen P, Richards SJ, et al. Sustained response and long-term safety of eculizumab in paroxysmal nocturnal hemoglobinuria. Blood. 2005;106(7):2559-2565

21. Kelly R, Arnold L, Richards S, et al. The management of pregnancy in paroxysmal nocturnal haemoglobinuria on long term eculizumab. Br J Haematol. 2010;149(3):446450.

22. Sharma R, Keyzner A, Liu J, Bradley T, Allen SL. Successful pregnancy outcome in paroxysmal nocturnal hemoglobinuria ( $\mathrm{PNH}$ ) following escalated eculizumab dosing to control breakthrough hemolysis. Leuk Res Rep. 2015;4(1):36-38.

23. Kelly R, Richards S, Hillmen P, Hill A. The pathophysiology of paroxysmal nocturna hemoglobinuria and treatment with eculizumab. Ther Clin Risk Manag. 2009, 5:911-921.

24. Risitano AM, Notaro R, Marando L, et al. Complement fraction 3 binding on erythrocytes as additional mechanism of disease in paroxysmal nocturnal hemoglobinuria patients treated by eculizumab. Blood. 2009; 113(17):4094-4100.

25. Hill A, Kelly RJ, Hillmen P. Thrombosis in paroxysmal nocturnal hemoglobinuria. Blood. 2013;121(25):4985-4996.

26. Lee JW, Jang JH, Kim JS, et al. Clinical signs and symptoms associated with increased risk for thrombosis in patients with paroxysmal nocturnal hemoglobinuria from a Korean Registry. Int J Hematol. 2013; 97(6):749-757.

27. Yenerel MN, Muus P, Wilson A, Szer J. Clinical course and disease burden in patients with paroxysmal nocturnal hemoglobinuria by hemolytic status. Blood Cells Mol Dis. 2017;65:29-34.

28. Brauckmann S, Effenberger-Neidnicht K, de Groot $\mathrm{H}$, et al. Lipopolysaccharide-induced hemolysis: evidence for direct membrane interactions. Sci Rep. 2016;6:35508.

29. Quist J, Hill AR. Serum lactate dehydrogenase (LDH) in Pneumocystis carinii pneumonia, tuberculosis, and bacterial pneumonia. Chest. 1995;108(2):415-418.

30. Oner AF, Bay A, Arslan S, et al. Avian influenza A (H5N1) infection in eastern Turkey in 2006. N Engl J Med. 2006;355(21):2179-2185.

31. Erez A, Shental O, Tchebiner JZ, et al Diagnostic and prognostic value of very high serum lactate dehydrogenase in admitted medical patients. Isr Med Assoc J. 2014 16(7):439-443 